\title{
Are supergranule sizes anti-correlated with magnetic activity?
}

\author{
N. Meunier, T. Roudier, and R. Tkaczuk
}

\author{
Laboratoire d'Astrophysique de l'Observatoire Midi-Pyrénées, Université Paul Sabatier, CNRS, 57 avenue d'Azereix, BP 826, \\ 65008 Tarbes Cedex, France \\ e-mail: [meunier; roudier]@ast.obs-mip.fr
}

Received 26 October 2006 / Accepted 23 January 2007

\section{ABSTRACT}

\begin{abstract}
Context. The variation of supergranule cell sizes with the magnetic environment is still controversial. Aims. We study this relation in detail to understand the discrepancies observed between previous results. Methods. We determine the cell size using divergence of horizontal flows derived from local correlation tracking of intensity maps (MDI/SOHO). We study the variation of the cell size as a function of the magnetic field inside the cell. We also consider which component of the magnetic field most influences the cell size.

Results. Our main conclusion is that there are no large cells when the magnetic field (in absolute value) averaged over the cell is large. This is mostly due to the magnetic field inside the cell (intranetwork fields), while strong network magnetic fields (at the cell boundary) are associated with larger cells. Further studies of the evolution of the cells and of the flux imbalance suggest that a high level of weak fields may prevent the formation of large cells. This is compatible with the expectation that strong magnetic fields should prevent large-scale flows.

Conclusions. The relation between the local activity level determined by the average magnetic field inside the cells and the supergranule size is not linear. Furthermore, it strongly depends on the definition of the activity level (magnetic field inside the cell or magnetic network) and on the magnetic sensitivity of the data. This last point probably explains at least partially the conflicting results obtained up to now.
\end{abstract}

Key words. Sun: activity - Sun: magnetic fields - Sun: granulation - Sun: photosphere - Sun: general

\section{Introduction}

The variation of granule sizes with the magnetic environment is well known (Schmidt et al. 1988; Muller et al. 1989; Title et al. 1992). However, it is quite controversial for supergranules as some authors (Table 1) found an increase in size with increasing activity, while others found either a decrease or no variation at all. The same controversy stands for the variation of supergranule sizes during the solar cycle. Table 1 gives a summary of the previous results obtained using two different approaches, namely the variation with the magnetic field at a given time over the field-of-view (spatial variation) and the variation during the solar cycle (temporal variation). In all previous works except that of DeRosa \& Toomre (2004), the determination of the cells themselves or of the cell sizes has been made using the magnetic network, either directly from photospheric magnetograms or indirectly from Ca II K images (chromospheric level). Therefore the cell size determination and the activity level characterization were not determined independently. This is unfortunate as it can lead to some biases. For example, if the magnetic field inside a cell is large (which is likely to happen when the average activity level is larger), there is a risk to split that cell into at least two parts, which would lead to smaller cells. It is therefore easy to artificially bias the result toward smaller cells when the activity level is higher. The magnetic sensitivity of the data could also bias the results. The results of DeRosa \& Toomre (2004), which have been obtained by tracking Doppler features, is probably the most reliable as the cell size determination is not made using data dominated by magnetic fields. However, they only give the average cell size for two periods, one being more active than the other, and do not study in detail the dependence of the cell sizes on the local magnetic field.

To address this long-standing question, we have determined supergranule cells using divergence of the horizontal flow velocity as in Meunier et al. (2007a,b). These measurements are independent of the magnetic fields. We will study the variation of the cell size with the magnetic field inside the cells at a given time (namely the solar cycle minimum). To get a complete picture, we also study the precise location inside the cells of the magnetic field influencing the cell size variations. We will show that the definition of the activity level is crucial, as it will be necessary to determine which component of the magnetic field influences the cell size variations. It will also be of interest to consider the temporal evolution of the cells: is the observed variation due to different initial condition for small and large cells, or is it the result of the development of the cells?

The data and processing are briefly described in Sect. 2. In Sect. 3, we study the variation of the cell size with the activity inside the cells, first by considering the magnetic field averaged over the cells or part of the cells, then by considering the magnetic field for each pixel of the images. In Sect. 4, we address the causality link leading to the observed relationship, through the study of the cell evolution and the degree of mixity between positive and negative magnetic fields. Finally, in Sect. 5, we study the dependence of the result on the magnetic sensitivity, which may provide an explanation for the incoherent results obtained in previous works. We discuss the results and conclude in Sect. 6. 
Table 1. List of variations of supergranule sizes with the magnetic field from the literature. The first 5 references concern studies at a given time (spatial variations), while the last 6 references concern studies covering the solar cycle or part of it (temporal variations). FT means Fourier transform. The sign "+" means an increase of the cell size with increasing activity level, while the sign "-" means the opposite variation and "0" no variation.

\begin{tabular}{clccc}
\hline \hline Reference & Data & Method & Variation & Comments \\
\hline Sýkora (1970) & Ca II K images & autocorrelation & + & Large errorbars \\
Wang (1988) & magnetograms & autocorrelation & + & \\
Wang et al. (1996) & magnetograms & autocorrelation & + & Via latitude variations \\
Hagenaar et al. (1997) & Ca II K images & segmentation & 0 & \\
Raju \& Singh (2002) & Ca II K images & autocorrelation & - & \\
\hline Sýkora (1970) & CaII K images & autocorrelation & + & \\
Singh \& Bappu (1981) & Ca II K images & autocorrelation? & - & FWHM of the autocorrelation curves \\
Muenzer et al. (1989) & Ca II K images & 2D TF & + & Over 1 year only \\
Komm et al. (1995) & magnetograms & autocorrelation & + & F time series \\
Berrilli et al. (1999) & Ca II K images & segmentation & - & - \\
DeRosa \& Toomre (2004) & Doppler & segmentation & - &
\end{tabular}

\section{Data processing}

We use high resolution MDI/SOHO (Scherrer et al. 1995) intensity maps and magnetograms obtained during the solar minimum, in January 1997 and March 1997. The pixel size is 0.605 arcsec and the field-of-view $620^{\prime \prime} \times 303^{\prime \prime}$. The temporal cadence is $1 \mathrm{~min}$. We define hourly data sets, each containing 60 raw images. 61 such hourly data sets are available in our sample (46 in January 1997 and 15 in March 1997).

For each hourly data set, the 60 intensity maps and magnetograms are aligned with the map at the center of the data set. Magnetograms are averaged together to provide a single low-noise magnetogram per hour. Intensity maps are corrected for large-scale gradients and $k-\omega$ filtered. A local correlation tracking algorithm is then applied to provide velocity and divergence maps. These divergence maps are averaged over $1 \mathrm{~h}$ and smoothed with a Gaussian whose full-width at half-maximum is $10.2 \mathrm{Mm}$ to exhibits supergranular cells. The validity of this approach is discussed in Meunier et al. (2007b).

The divergence maps are used for two purposes. First, a steepest descent algorithm is applied (see Hagenaar et al. 1997; Meunier et al. 2007a,b) to determine the boundary of the cells. This leads to a cell size defined by the maximum radius of the cell, hereafter $R$. The smoothed divergence maps also provide a way to determine the relative distance to cell center in each cell, noted $d_{\text {rel }}$. The cell center corresponding to $d_{\text {rel }}=0$, is defined as the barycenter of the cell. Furthermore, a normalized smoothed divergence, noted $D_{\text {norm }}$, is defined. It takes the value 0 at the location of maximum positive divergence in the cell (which in general does not coincide exactly with $d_{\text {rel }}=0$, although $d_{\text {rel }}$ and $D_{\text {norm }}$ are correlated), 0.5 at positions of zero divergence and 1 at position of maximum convergence. This normalized divergence provides an alternative way to take into account the localization of the magnetic field inside the cells. We refer to Meunier et al. $(2007 a, b)$ for more details about the processing.

\section{Variation of the cell size with the activity level}

We will use two different approaches. In the first one, we consider properties averaged over the cell: for each cell, we consider the size $R$ and the absolute value of the magnetic field $\left|B_{\mathrm{c}}\right|$ averaged over the cell. A complementary analysis is to average $|B|$ over a specific part of the cell (for example close to the cell center or close to the cell boundary), using selections based on $d_{\text {rel }}$ or $D_{\text {norm }}$, to study which component of the magnetic field influences the cell size. This magnetic field is denoted $\left|B_{\mathrm{r}}\right|$.
Another approach will be to consider the absolute value of the magnetic field for each pixel, $\left|B_{\mathrm{p}}\right|$, and then to associate the size $R$ of the cell to which the pixel belongs. This will be very useful to study for example the magnetic field variations inside the cells for various cell size ranges, i.e. radial profiles of the magnetic fields inside the cells.

The magnetic field we are considering is the line-of-sight component. A small projection effect is therefore present. For the magnetic network for example, expected to be mainly vertical, values in the corners of our fields of view should be smaller by a few percent than at disk center: $\cos (\theta)$ ranges from 1 at the center of the images to 0.95 in the corners. $90 \%$ of the pixels have values above $0.96,61 \%$ above 0.98 . This effect can however be neglected in what follows, as is the influence of the projection effect on the supergranule size, since the observed variations are larger than a few percent (see for example Figs. 2 or 3 ).

\subsection{Magnetic properties averaged over the cells}

\subsubsection{Averages over the entire cell}

We first consider properties averaged over each cell or over part of each cell. Figure 1 shows the cell size $R$ versus the absolute value of the magnetic field averaged over the cell, $\left|B_{\mathrm{c}}\right|$. The solid curve (left panel) gives the mean cell size for each magnetic field bin. The errorbars are the uncertainties on the mean at the $1 \sigma$ level, as for the other similar curves shown in this paper. A small decrease of the cell size is observed as the magnetic field in the cell increases, except for the smallest sizes and magnetic fields. However, this decrease seems to be due to the fact there is no large cell with a strong magnetic field rather than to an actual shift of distribution, as shown in the 1D histograms of Fig. 1 (middle and right panels). When considering the distribution of $R$ values for various ranges of $\left|B_{\mathrm{c}}\right|$ for example, the tail of the distribution is smaller for strong magnetic fields, while the peak of the distribution correspond to an almost constant $R$. In the left panel, the solid curve shows a variation of the cell size on the order of $4 \mathrm{Mm}$, while the position of the peak of the distributions (middle panel) does not change by more than $1 \mathrm{Mm}$.

We also note that the left panel of Fig. 1 presents strong similarities with the scatter plot shown by Hagenaar et al. (1997) in their Fig. 9. They concluded that there was no variation of the cell size with the activity level, but in fact the distributions show that there is indeed a variation. This difference will also 

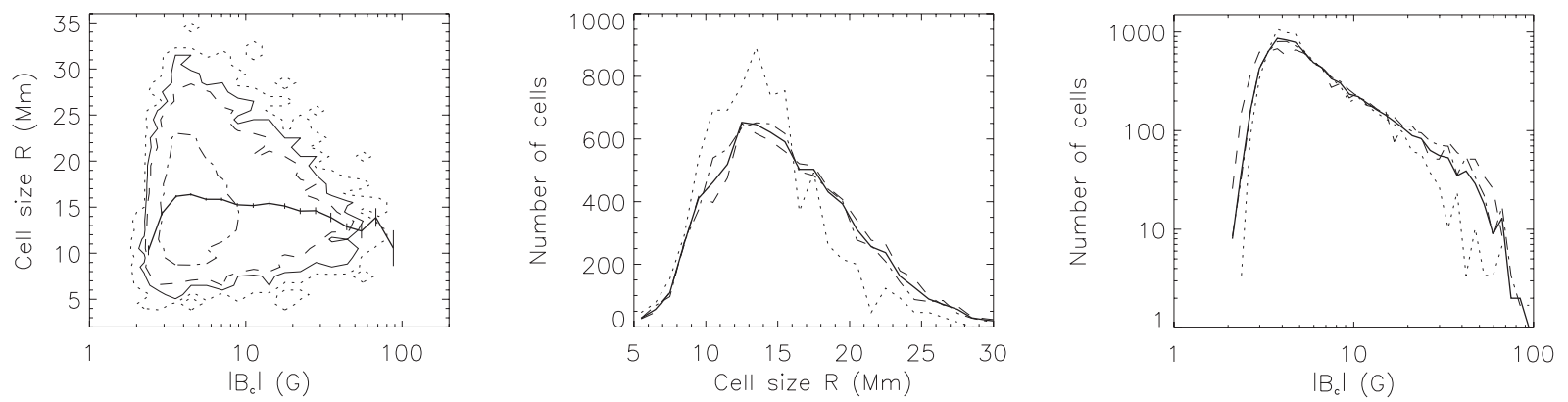

Fig. 1. Left: number of cells as a function of the absolute value of the magnetic field averaged over the cell $\left|B_{\mathrm{c}}\right|$ and of the cell size $R$. Contours are at the levels of 1 cell (dotted line), 5 (solid line), 10 (dashed line), and 50 (dotted-dashed line) cells. The thick solid line represents the average cell size in small $\left|B_{\mathrm{c}}\right|$ ranges. Middle: number of cells as a function of $R$, for various ranges of $\left|B_{\mathrm{c}}\right|$ averaged over the cell: all cells (thick solid line), cells with $\left|B_{\mathrm{c}}\right|$ smaller than $10 \mathrm{G}$ (dashed line), cells with $\left|B_{\mathrm{c}}\right|$ in the range $10-18 \mathrm{G}$ (dotted-dashed line) and cells with $\left|B_{\mathrm{c}}\right|$ larger than $18 \mathrm{G}$ (dotted line). Histograms are normalized by the number of cells used to build the thick curve. Right: number of cells as a function of $\left|B_{\mathrm{c}}\right|$ averaged over the cell, for various size ranges: all cells (thick solid line), cells with $R$ smaller than $6 \mathrm{Mm}$ (dashed line), cells with $R$ in the range 6-20 Mm (dotted-dashed line) and cells with $R$ larger than $20 \mathrm{Mm}$ (dotted line). Histograms are normalized by the number of cells used to build the thick curve.
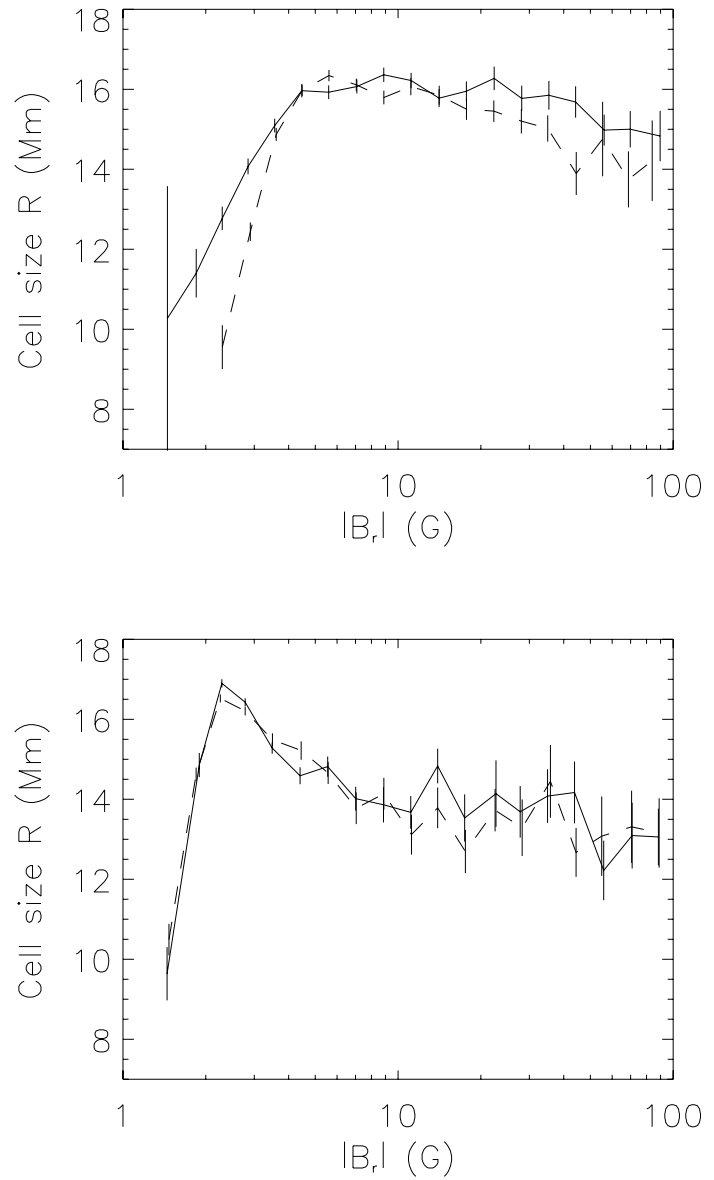

Fig. 2. Cell size $R$ versus the absolute value of the magnetic field $\left|B_{\mathrm{r}}\right|$ averaged over various regions in each cell. Upper panel: in converging flows (solid line) and at cell boundary (dashed line). Lower panel: in diverging flows (solid line) and at cell center (dashed line).

be discussed in more detail in Sect. 5 as the flatter curve they observe may be due to the magnetic sensitivity.

\subsubsection{Averages over particular areas of the cells}

Our goal is now to understand the origin of this variation. To investigate this variation in more detail, we have computed the
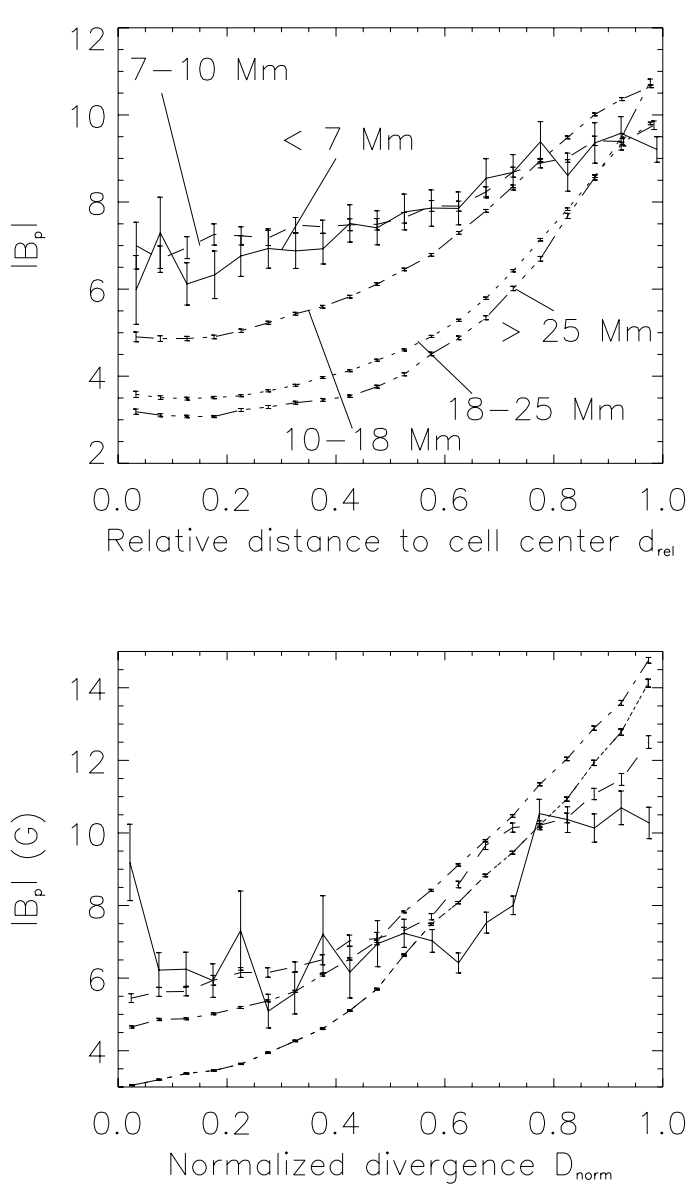

Fig. 3. Upper panel: absolute value of magnetic field at each pixel $\left|B_{\mathrm{p}}\right|$ versus the relative distance to cell center $d_{\text {rel }}$, for various size ranges: $R$ lower than $7 \mathrm{Mm}$ (solid line), in the range 7-10 Mm (dashed line), in the range 10-18 Mm (dotted-dashed line), in the range 18-25 Mm (dotted line), and larger than $25 \mathrm{Mm}$ (dot-dot-dot-dashed line). Lower panel: same versus the normalized smoothed divergence $D_{\text {norm }}$.

average magnetic field in various parts of the cell, $\left|B_{\mathrm{r}}\right|$ : in the strong diverging flows $\left(D_{\text {norm }}\right.$ smaller than 0.2$)$, in the strong converging flows $\left(D_{\text {norm }}\right.$ larger than 0.8$)$, at cell center $\left(d_{\text {rel }}\right.$ smaller than 0.2$)$ and at cell boundary $\left(d_{\text {rel }}\right.$ larger than 0.8$)$. The variation of the cell size as a function of these new estimates of the magnetic field is shown in Fig. 2. 
Two regimes are observed. First, for very small magnetic fields, we note that very weak field regions are associated with the smallest cells. This could be seen in Fig. 1, although less clearly. The amplitude of this variation does not seem to depend much on the localization of the magnetic field in the cell. However, the position of the maximum of the curves depends on the localization and varies between $3 \mathrm{G}$ (diverging flows and cell center) and $7 \mathrm{G}$ (converging flows and cell boundary). In the first case the peak in the curve is quite sharp and the uncertainty on the $3 \mathrm{G}$ value is smaller than $1 \mathrm{G}$. In the second case, the maximum is well defined around $7 \mathrm{G}$ for the cell boundary, with similar uncertainties, and it is rather a plateau starting at $7 \mathrm{G}$ for converging flows. There is great similarity between curves for diverging flows and cell center. The same is true for converging flows and cell boundary curves.

Then a second regime, which concerns most of the cells, is observed for magnetic fields larger than a few Gauss and corresponds to the observation already discussed in Sect. 3.1.1. Here, we also observe a decrease of the cell size as the magnetic field increases. However, the amplitudes of the variations are different. A rough estimate from Fig. 2 shows that the strongest variation is observed for the magnetic field estimated in the diverging flows (variation of $\sim 4 \mathrm{Mm}$ ) and in the cell center ( $\sim 3 \mathrm{Mm})$, followed by the variation at the cell boundary $(\sim 2 \mathrm{Mm})$ and in the converging flows $(\sim 1 \mathrm{Mm})$.

The magnetic field at the cell boundary is well correlated with the magnetic field in converging flows (0.67) as well as the field at cell center with the field in diverging flows (0.66), which is expected. However, the correlation between the field at cell center and at cell boundary is much smaller (0.33) and it is even worse between the field in diverging flows and converging flows (0.17). This shows that to define an "activity level", even local, is not that easy. Which component of the magnetic field are we talking about? Do they have the same influence? We notice that the behavior for $\left|B_{\mathrm{r}}\right|$ defined at cell center or at the boundary does not give the same result.

\subsection{Radial profiles and magnetic field distributions inside cells}

Given the results obtained when averaging the magnetic field in specific parts of the cells, we study further the dependence on the localization. Therefore, we now consider all the pixels of the maps. To each pixel we associate the size of the cell to which it belongs. We first consider the profiles of the magnetic field as a function of the relative distance to the cell center $d_{\text {rel }}$ and as a function of the normalized divergence $D_{\text {norm }}$, and then the distribution of the magnetic field values for various size ranges. $\left|B_{\mathrm{p}}\right|$ denotes the absolute value of the magnetic field at each pixel. These profiles are an extension of a "radial profile", as it is not only a geometrical definition (using $d_{\text {rel }}$ ), but also correspond to a definitioni directly using the flows ( $\left.D_{\text {norm }}\right)$.

\subsubsection{The magnetic field radial profiles inside the cell}

We consider the profiles of $\left|B_{\mathrm{p}}\right|$ versus the relative distance to cell center $d_{\text {rel }}$ and versus the normalized divergence $D_{\text {norm }}$, for various cell size ranges. The results are shown in Fig. 3. Close to the cell center, a very strong decrease in magnetic field is observed as the cell size increases, and this is seen up to values of $d_{\text {rel }}$ around 0.7. On the other hand, a small increase in magnetic field is observed at the cell boundary, for $d_{\text {rel }}$ above 0.9 . When considering the variation with the normalized divergence $D_{\text {norm }}$,

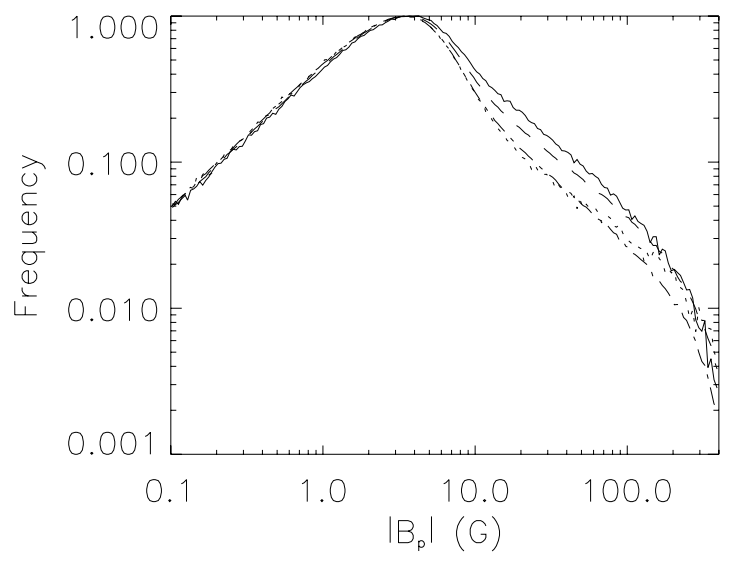

Fig. 4. Distribution of the absolute value of magnetic field at each pixel $\left|B_{\mathrm{p}}\right|$ in each pixel for different size ranges: $R$ lower than $10 \mathrm{Mm}$ (solid line), in the range 10-16 $\mathrm{Mm}$ (dashed line), in the range 16-20 Mm (dotted-dashed line) and larger than $20 \mathrm{Mm}$ (dotted line). The histograms are normalized to their maximum.

we also observe a strong decrease of the magnetic field in diverging flows, for $D_{\text {norm }}$ up to $\sim 0.3$. However, above 0.5 , the trend is reversed again, and the magnetic field increases as the cell size increases. This confirms that the variation in cell size is mostly dependent on the magnetic field inside the cell. There may also be a reversal at the cell boundary due to the magnetic network. It can indeed be expected that strong magnetic field network can be present only when the convergences are strong, in large and long-lived cells (DeRosa \& Toomre 2004). The reversal is stronger for $D_{\text {norm }}$ than for $d_{\text {rel }}$, which is expected as the magnetic network exhibits a better correlation with the regions of strong convergence than with the entire boundary of the cells. Therefore this provides a coherent picture.

\subsubsection{The magnetic field distributions}

Finally, we consider the magnetic field distribution for the whole surface, and when selecting specific regions inside the cells. The total distribution of $\left|B_{\mathrm{p}}\right|$ for various cell size ranges is shown in Fig. 4. The tail is shorter for large cells, as expected. Figure 5 now shows the same plots when considering the different categories of localization of the magnetic field in the cells: in strong diverging flows $\left(D_{\text {norm }}\right.$ smaller than 0.2), in strong converging flows $\left(D_{\text {norm }}\right.$ larger than 0.8$)$, at cell center $\left(d_{\text {rel }}\right.$ smaller than 0.2$)$ and at the cell boundary $\left(d_{\text {rel }}\right.$ larger than 0.8$)$. The tail is smaller for diverging flows and for the cell center, which is expected as there is almost no magnetic network there. The tail of the magnetic field distribution is again reduced when the size increases, at the cell center and in diverging flows. In converging flows, the longest tail is for the largest cells, confirming the reversal observed in Fig. 3.

\section{Evolution of the magnetic field in the cells, flux imbalance, and cell size}

In the previous section, we have shown that a strong magnetic field inside the cells is associated with smaller cells, while the dependence on the magnetic field at the boundary (mostly magnetic network) is reversed. As the magnetic field inside the cells increases, the possible maximum value for $R$ decreases, as was shown in Fig. 1. The main variation is then mostly a variation in 

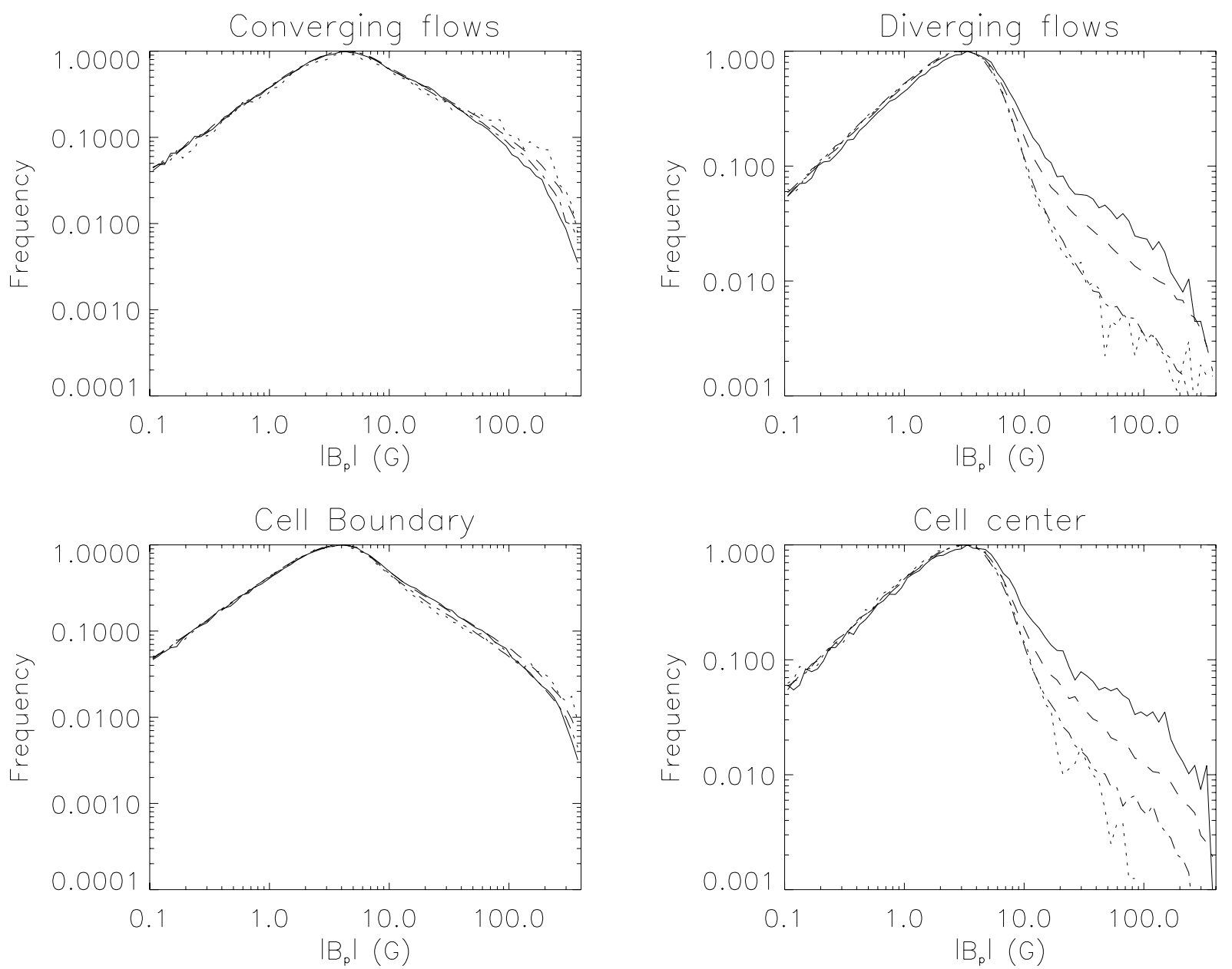

Fig. 5. Distribution of the magnetic field $\left|B_{\mathrm{p}}\right|$ in each pixel for different size ranges: $R$ lower than $10 \mathrm{Mm}$ (solid line), in the range $10-16 \mathrm{Mm}$ (dashed line), in the range 16-20 Mm (dotted-dashed line) and larger than $20 \mathrm{Mm}$ (dotted line). The magnetic field is considered in different regions of the cells: in converging flows (upper left panel), in diverging flows (upper right panel), at cell boundary (lower left panel), and at cell center (lower right panel). The histograms are normalized to their maximum.

the tail of the distributions. $R$ and $\left|B_{\mathrm{c}}\right|$ are here defined at a given time during the cell lifetime.

Two interpretations are possible: (i) in regions with weak magnetic fields, it is possible to have large cells while strong magnetic fields prevent the formation of large cells; or (ii) in large cells the polarities cancel each other faster, with a more efficient concentration of magnetic fields, resulting in a smaller flux, and in a more concentrated field at the boundary of the cells. However, if large cells form preferentially in unipolar regions, the more concentrated field at the boundary would not be associated with a smaller flux, but there is currently no indication that this is the case. The first hypothesis may be examined by studying the evolution of $B$ as a given cell evolves. The second hypothesis will be investigated by looking at the flux imbalance as a function of cell sizes as well as as by comparing this to the expected flux imbalance as a function of scale, independently of the cell determination.

\subsection{Evolution of the cells}

The question to answer is: when a cell is formed and increases in size to lead to a large cell, how does the magnetic field in that cell evolve? Is it weak at the beginning and then forms more concentrated features? Is it already larger at the beginning? It would be interesting to know if the results are related to the evolution of the cells (i.e. it is natural that when a small cell evolves toward a larger cell, the magnetic field decrease), or if they are related to their initial condition. Among small cells, there should be a large proportion of cells that remain small, and a few that lead to large cells. Thus, are the seeds of large cells found in regions with particularly weak magetic fields or not?

We have tracked supergranules as described in Meunier \& Roudier (2007). This tracking is a combination of local correlation and feature tracking, as the local correlation is computed only at places where the divergence is maximum: we therefore track the positions of maximum divergence. From this analysis, we derive a list of structures that are tracked in time, and we also know their properties at any time during their lifetime (provided that their first identification is not on the first image of the series). The magnetic field at cell center (or in diverging flows) tends to decrease over time for long-lived structures. However, the magnetic field computed at their starting time (first identification) shows a larger variation, which shows that the differences already exist when the cell is first identified (at least at the temporal resolution of $1 \mathrm{~h}$ ). We also note that long-lived structures have weaker magnetic fields inside the cells compared to shortlived cells, and have a constant field inside the network. This contradicts the results of DeRosa \& Toomre (2004), who concluded that cells embedded in a more active region tend to live longer, which was interpreted as a possible stabilisation effect of 


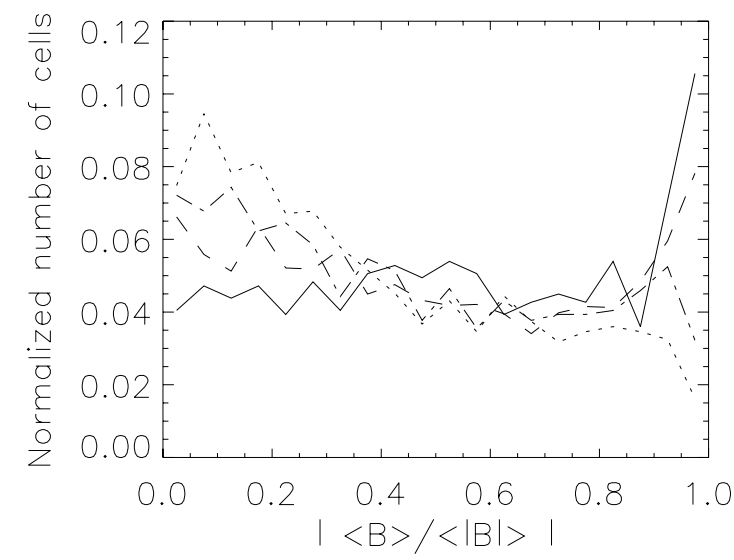

Fig. 6. Distribution of the flux imbalance $|\langle B\rangle /\langle|B|\rangle|$ for different size ranges: $R$ lower than $10 \mathrm{Mm}$ (solid line), in the range $10-16 \mathrm{Mm}$ (dashed line), in the range 16-20 Mm (dotted-dashed line) and larger than $20 \mathrm{Mm}$ (dotted line).

the magnetic field on the cells: from our analysis, this may not be the case.

A complete study of the evolution of cells is beyond the scope of the present paper and will be the subject of a future work. We can already conclude however that the evolution of the magnetic field inside the cells during their life is probably not at the origin of the observed relation between size and magnetic field in supergranular cells.

\subsection{Flux imbalance}

Let us consider the evolution of cells toward small or large cells starting from an initial distribution of the magnetic field. For a similar degree of magnetic field mixity and of average field strength, if the magnetic field in large cells tends to concentrate in strong magnetic field structures at the boundary, thus "emptying" the cells, one expects the magnetic field in these cells to be more unipolar, as more flux of opposite polarities will also cancel out. To check this, we computed the absolute value of the flux imbalance $|\langle B\rangle /\langle|B|\rangle|$ over each cell. Values around 0 mean a mixed polarity field, while values close to 1 mean unipolar field (of either polarity). Figure 6 shows the distribution of this imbalance for various size ranges. We observe that large cells are more often close to mixed polarity than smaller cells, while small cells are more often close to unipolar magnetic fields. Therefore we do not observe a larger unipolarity of the magnetic fields in large cells. This means that the initial conditions leading to cells of various sizes were probably different in field strength, in mixity, or both.

We compare these results with the imbalance computed over boxes of various sizes, independently of any image segmentation. We have chosen square boxes with size 16, 32, 64, 128 and 256 pixels, corresponding to $6.0 \mathrm{Mm}$ for the smallest size and $111 \mathrm{Mm}$ for the largest size. The value of 64 pixels is close to the supergranular size $(27.7 \mathrm{Mm})$. Boxes are distributed regularly over the surface, so that for a given size, we pave the whole surface with adjacent boxes of that size. The imbalance is computed for various thresholds of the magnetic field, i.e. only magnetic fields larger than a certain value are considered. For high thresholds, this provides the mixity associated with magnetic network only, while the low thresholds also take into account the intranetwork magnetic field observed here down to the noise level of $\sim 3 \mathrm{G}$. Figure 7 shows the magnetic threshold

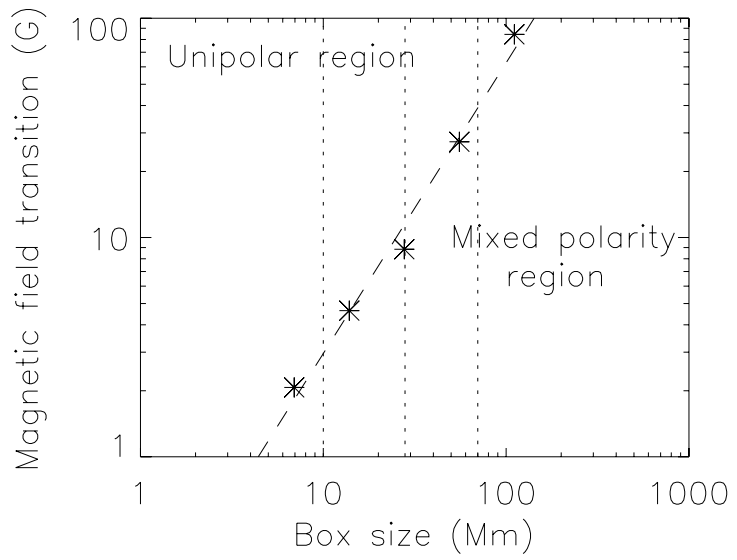

Fig. 7. Magnetic field threshold (stars) corresponding to an average flux imbalance of 0.5 , versus the size of the box over which the imbalance is computed. The dashed line is a linear fit. The vertical dotted lines indicate the minimum and maximum size of detected cells $(2 R)$ studied here as well as the size corresponding to the peak of their distribution.

for which the imbalance is around 0.5 . The unipolarity seems stronger for high magnetic thresholds. The limit between the two regimes increases with the box size. In the supergranular regime, the threshold is in the range $2-40 \mathrm{G}$, and is typically around $10 \mathrm{G}$ for a supergranule of $30 \mathrm{Mm}$. At a given threshold $\left|B_{\mathrm{th}}\right|$, more mixed polarity is observed for large scales. This is in good agreement with the imbalance computed as a function of the cell size. The two approaches are independent: the spatial distribution of the cells is probably close to random as the field-of-view corresponds to quiet sun (no active region to bias the distribution), while the square boxes cover the whole field of view and are therefore not biased. A possible explanation would be that the flux imbalance at the scale of the cell is not controled by the supergranular diffusion only, but also at a more global level, depending on large-scale motions such as the meridional circulation and on the distribution of active regions.

Note that the spatial resolution of the observation is important for this kind of study, because at low resolution, some flux is missed. Such a study should therefore be completed using largescale observations made with very high resolution such as the one that will be performed by the CALAS ${ }^{1}$ instrument at the Lunette Jean Rösch of the Pic du Midi Observatory (Meunier et al. 2003, 2005).

\section{Influence of the magnetic sensitivity on the results}

Our results could explain why contradictory results have been obtained in the past. If we consider good sensitivity magnetograms such as the one we are using here, we observe that no large cells can exist for strong magnetic fields, while there is a reversal when considering the magnetic field at the cell boundary (magnetic network). With a different sensitivity, we may be more sensitive to the influence of the magnetic network for example. We are now quantifying this effect.

As an indicator of the dependence, we compute the slope (derived from a linear fit on the scatter plot) of the cell size $R$ versus $\left|B_{\mathrm{c}}\right|$ and $\left|B_{\mathrm{p}}\right|$ in various cases. The slope of $\left|B_{\mathrm{c}}\right|$ and $\left|B_{\mathrm{p}}\right|$ versus $R$ is also computed for comparison. Instead of using all pixels in the cells, we consider the pixels with $\left|B_{\mathrm{p}}\right|$ larger than a

\footnotetext{
${ }^{1}$ CAmera for the LArge Scales of the solar surface.
} 

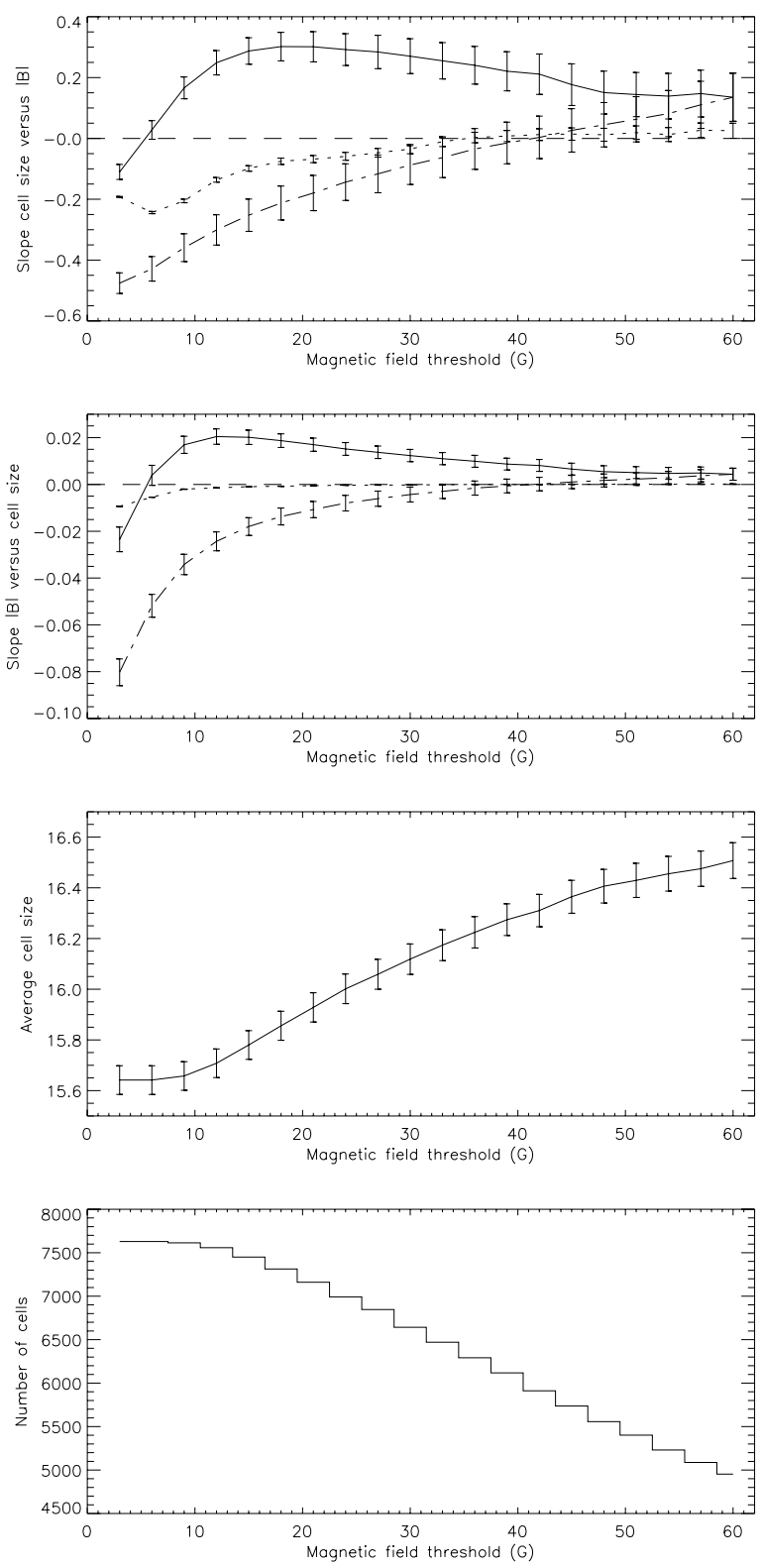

Fig. 8. First panel: slope of the cell size $R$ versus the magnetic field $|B|$ as a function of the threshold $B_{\mathrm{th}} .|B|$ is chosen larger than $B_{\mathrm{th}}$. Plots are for cells such that $\left|B_{\mathrm{c}}\right|$ is larger than $B_{\text {th }}$ (solid) and for cells such that $\left|B_{\mathrm{c}}\right|$ is larger than $60 \mathrm{G}$ (dashed line). The dotted line corresponds to the computations made on all pixels with $\left|B_{\mathrm{p}}\right|$ larger than $B_{\mathrm{th}}$. Second panel: same for the slope of the magnetic field $|B|$ versus the cell size $R$. Third panel: average cell size for each selection (cells such that $\left|B_{\mathrm{c}}\right|$ is larger than $B_{\text {th }}$ ). Fourth panel: number of cells for each selection (cells such that $\left|B_{\mathrm{c}}\right|$ is larger than $\left.B_{\mathrm{th}}\right)$.

certain threshold $\left|B_{\text {th }}\right|$, to simulate observations of various magnetic sensitivities. As some cells may not have any pixels with a magnetic field larger than the threshold, the sample is different for various $\left|B_{\mathrm{th}}\right|$ : at larger thresholds, some cells are eliminated from the computation. Therefore, we have also considered the variation when considering the same sample, i.e. the sample for the largest threshold considered $(60 \mathrm{G})$.

The results are shown in Fig. 8 as well as the average cell size and the number of cells for each sample. When using the same sample for all $\left|B_{\mathrm{th}}\right|$, we observe a negative slope for small $\left|B_{\mathrm{th}}\right|$ (as observed in Sect. 3). The sign of the slope changes around $40 \mathrm{G}$ and then becomes positive. When selecting a different sample (i.e. the sample containing all cells for which the computation can be done), the reversal takes place much sooner, for $\left|B_{\text {th }}\right|$ around $5 \mathrm{G}$. For comparison, the slope for $\left|B_{\mathrm{p}}\right|$ shows intermediary values. The differences are due to the different weightings of cells of different properties in the two approaches. With $\left|B_{\mathrm{p}}\right|$, more weight is given to cells with more pixels larger than the threshold. The size of the sample is also intermediate in the case of $\left|B_{\mathrm{p}}\right|$, since some cells may have a $\left|B_{\mathrm{c}}\right|$ lower than $\left|B_{\mathrm{th}}\right|$ but may contain pixels with $\left|B_{\mathrm{p}}\right|$ larger than $\left|B_{\mathrm{th}}\right|$.

The difference between the two types of sampling can be understood with Fig. 9, which shows the number of cells versus $R$ and $\left|B_{\mathrm{c}}\right|$ simultaneously for the same sampling and a different sampling for four examples of $\left|B_{\mathrm{th}}\right|$. For small $\left|B_{\mathrm{th}}\right|$ (upper left panel), we see that the cells removed when restricting the sampling are mostly very small cells with small magnetic fields (cells in the lower left section of the panel), so it is easy to understand why the slope is closer to zero or is reversed (as shown by the two curves showing the mean magnetic field in each cell size bin). When increasing the threshold, the shape of the distribution is changed, and the limitation of large cells at strong magnetic fields seems less present. The slopes are only an indicator, as the relation between the cell size and the average magnetic field over the cell is not linear, and in all cases the distributions must be considered to get a clear picture, as the correlation between $R$ and $\left|B_{\text {th }}\right|$ is small.

Depending of the magnetic sensitivity of the observations, the weight given to the magnetic network and to the magnetic field inside the cells will be different, providing different dependences between $R$ and $\left|B_{\text {th }}\right|$. Furthermore, the magnetic field inside the cells may be missed for various reasons. For example, Hagenaar et al. (1997) have averaged their Ca II K images over several hours, and these weak field structures may then not be seen if their lifetime is not that long.

\section{Conclusion}

We have studied in detail the dependence of the supergranule size as a function of the local activity level in the cell. This is the first time that this work is performed with a determination of the cells independent of the magnetic field measurement, since previous authors have used either magnetograms or $\mathrm{CaK}$ images to identify the cell size, either by segmentation or by autocorrelation. An exception is the work of DeRosa \& Toomre (2004) on Dopplergrams: they only give the average cell size for two periods, and do not study in detail the relationship between the activity level and the cell size.

We conclude that there are two answers to the original question: are supergranule sizes anti-correlated with magnetic activity? Our main conclusion is that there are no large cells when the average magnetic field (in absolute value) in the cell is large. This is mostly due to the magnetic field inside the cell (intranetwork fields), while strong network magnetic fields are associated with larger cells. Further studies of the evolution of the cells and of the flux imbalance suggest that the main explanation could be that high levels of weak fields may prevent the formation of large cells. This is compatible with the expectation that strong magnetic fields should prevents large scale flows, as observed for granules. This conclusion will have to be confirmed with studies at a higher temporal resolution.

We conclude that there is not a single answer to the longstanding question concerning the dependence of supergranule size on the activity level, as it strongly depends on the magnetic field definition. This also explains the apparent contradiction between previous results. It also means that the study of the 

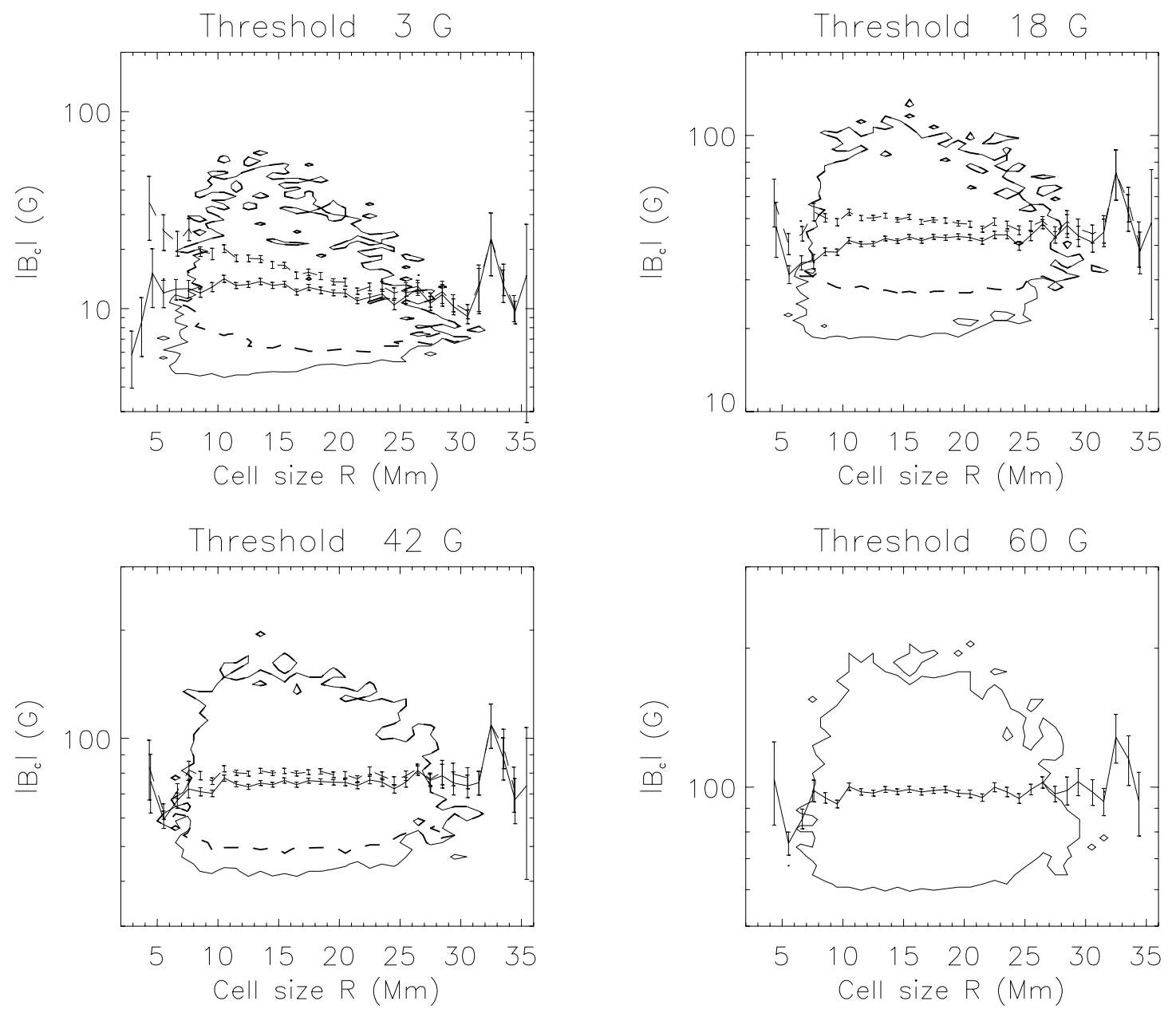

Fig. 9. Contour of the distribution of the magnetic field $\left|B_{\mathrm{c}}\right|$ in the cell versus the cell size $R$, for 4 different magnetic field lower thresholds $B_{\mathrm{th}}$. The thick solid line represents the contour for all cells with $|B|$ larger than the current threshold, while the thick dashed line represents the contour for a subset of cells corresponding to the $60 \mathrm{G}$ threshold. The two thin curves with errorbars show the mean magnetic field for each cell size bin, for the subset corresponding to the current threshold (solid line) and for the subset corresponding to the $60 \mathrm{G}$ threshold (dashed line). The errorbars show the uncertainty on the mean at the $1 \sigma$ level.

possible variation of supergranule sizes during the solar cycle must carefully define the magnetic field. Such a study, which considers the activity level over the whole sun at the time of the observation and not the magnetic field strength inside the cell as done in the present work, will also provide complementary information that will help to interpret the relation between the activity level and the supergranule cell size.

An interpretation of the radial profiles inside the cells should also be provided by a comparison with the results of simulations of supergranulation in presence of magnetic fields. This remains to be done, as no simulation currently provides such profiles. The interpretation of these profiles is strongly related to the origin of the intranetwork and network magnetic fields. Depending on their origin, either generated by the global dynamo through active region remants or generated locally through a local dynamo action (e.g. Cattaneo et al. 2003), the resulting radial profiles may be different, and may have different correlations with the amplitude of the divergence in the cell. A complementary analysis using cork diffusion, similar to those performed by Krijger et al. (2002), should also be applied to these observations. This will be the subject of future work.

Acknowledgements. SOHO is a mission of international cooperation between the European Space Agency (ESA) and NASA.

\section{References}

Berrilli, F., Ermolli, I., Florio, A., \& Pietropaolo, E. 1999, A\&A, 344, 965 Cattaneo, F., Emonet, T., \& Weiss, N. 2003, ApJ, 588, 1183

DeRosa, M. L., \& Toomre, J. 2004, ApJ, 616, 1242

Hagenaar, H. J., Schrijver, C. J., \& Title, A. M. 1997, ApJ, 481, 988 Komm, R. W., Howard, R. F., \& Harvey, J. W. 1995, Sol. Phys., 158, 213

Krijger, J. M., Roudier, T., \& Rieutord, M. 2002, A\&A, 387, 672

Meunier, N., \& Roudier, T. 2007, A\&A, 466, 691

Meunier, N., Rieutord, M., \& Beigbeder, F. 2003, in SF2A-2003: Semaine de l'Astrophysique Française, ed. F. Combes, D. Barret, T. Contini, \& L. Pagani, 93

Meunier, N., Rondi, S., Tkaczuk, R., Rieutord, M., \& Beigbeder, F. 2005, in Large-scale Structures and their Role in Solar Activity, ed. K. Sankarasubramanian, M. Penn, \& A. Pevtsov, ASP Conf. Ser., 346, 53

Meunier, N., Tkaczuk, R., \& Roudier, T. 2007a, A\&A, 463, 745

Meunier, N., Tkaczuk, R., Roudier, T., \& Rieutord, M. 2007b, A\&A, 461, 1141 Muenzer, H., Schroeter, E. H., Wöhl, H., \& Hanslmeier, A. 1989, A\&A, 213, 431

Muller, R., Hulot, J. C., \& Roudier, T. 1989, Sol. Phys., 119, 229

Raju, K. P., \& Singh, J. 2002, Sol. Phys., 207, 11

Scherrer, P. H., Bogart, R. S., Bush, R. I., et al. 1995, Sol. Phys., 162, 129

Schmidt, W., Grossmann-Doerth, U., \& Schroeter, E. H. 1988, A\&A, 197, 306

Singh, J., \& Bappu, M. K. V. 1981, Sol. Phys., 71, 161

Sýkora, J. 1970, Sol. Phys., 13, 292

Title, A. M., Topka, K. P., Tarbell, T. D., et al. 1992, ApJ, 393, 782

Wang, H. 1988, Sol. Phys., 117, 343

Wang, H., Tang, F., Zirin, H., \& Wang, J. 1996, Sol. Phys., 165, 223 